\title{
Perfil de clientes vegetarianos de um restaurante universitário de Vitória-ES
}

\author{
Profile of vegetarian customers of a university restaurant of Vitória-ES \\ Perfil de clientes vegetarianos de un restaurante universitário de Vitória-ES
}

\section{Resumo}

O presente estudo teve como objetivo avaliar o perfil sociodemográfico, de hábitos alimentares e de conhecimentos em nutrição dos clientes vegetarianos de um restaurante universitário. Este é um estudo transversal realizado com 3348 clientes de um estabelecimento de alimentação coletiva vinculado a uma instituição de ensino superior de Vitória-ES, dos quais 196 eram vegetarianos. Utilizou-se um questionário semiestruturado com questões sobre perfil sociodemográfico, hábitos alimentares e conhecimentos relacionados à nutrição. Os dados foram analisados no SPSS, adotando-se $\mathrm{p}<0,05$. No total, os participantes eram predominantemente estudantes $(94,6 \%, \mathrm{n}=3166)$, sendo a mediana de idade de 21 anos. A prevalência do vegetarianismo foi maior entre os estudantes de cursos diferentes da área da saúde $(6,8 \%)$ e os do sexo feminino $(7,2 \%)$, sendo $\mathrm{p}<0,001$. Entre os vegetarianos $(\mathrm{n}=196)$, houve predomínio da classe de ovolactovegetarianos $(63,8 \% ; n=125)$ e os veganos representaram $10,2 \%(n=20)$. O motivo ético foi o mais mencionado entre os adeptos $(50,5 \%)$ e a maioria relatou procurar informações sobre a prática em fontes não científicas como sites da internet $(74,4 \%, n=134)$ e blogs $(25,6 \%, n=46)$. A pontuação referente ao conhecimento em

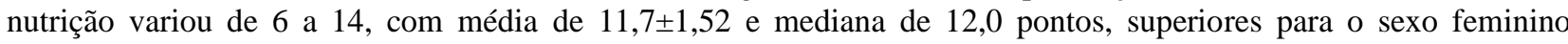
$(11,94 \pm 1,33, \mathrm{p}=0,006)$. O conhecimento do perfil de vegetarianos é fundamental para o atendimento das necessidades deste público.

Palavras-chave: Alimentação coletiva; Nutrição; Vegetarianismo.

\begin{abstract}
This study aimed to assess the sociodemographic profile, eating habits and knowledge of nutrition of vegetarian customers in a university restaurant. This is a cross-sectional study carried out with 3348 customers of a collective feeding establishment linked to a higher education institution in Vitória-ES, of which 196 were vegetarians. A semistructured questionnaire was used with questions about sociodemographic profile, eating habits and knowledge about nutrition. The data were analyzed in SPSS, adopting $\mathrm{p}<0.05$. In total, the participants were predominantly students $(94.6 \%, \mathrm{n}=3166)$, with a median age of 21 years. The prevalence of vegetarianism was higher among students from courses in areas other than health $(6.8 \%)$ and females $(7.2 \%), \mathrm{p}<0.001$. Among vegetarians $(\mathrm{n}=196)$, there was a predominance of ovolactovegetarian $(63.8 \% ; n=125)$ and vegans represented $10.2 \%(n=20)$. The ethical motive was the most mentioned among the adherents $(50.5 \%)$ and the majority reported looking for information about the practice in non-scientific sources such as internet sites $(74,4 \%, \mathrm{n}=134)$ e blogs $(25.6 \%, \mathrm{n}=46)$. The score referring to knowledge in nutrition ranged from 6 to 14, with a mean of $11.7 \pm .1 .52$ and median of 12.0 points, higher for females $(11.94 \pm 1.33, \mathrm{p}=0.006)$. The knowledge about the profile of vegetarians is essential to satisfy the needs of this public. Keywords: Food service; Nutrition; Vegetarianism.
\end{abstract}




\begin{abstract}
Resumen
Este estudio tuvo como objetivo evaluar el perfil sociodemográfico, los hábitos alimentarios y los conocimientos nutricionales de los clientes vegetarianos en un restaurante universitario. Se trata de un estudio transversal realizado con 3348 clientes de un establecimiento colectivo de alimentación vinculado a una institución de educación superior en Vitória-ES, de los cuales 196 eran vegetarianos. Se utilizó un cuestionario semiestructurado con preguntas sobre perfil sociodemográfico, hábitos alimentarios y conocimientos en nutrición. Los datos se analizaron mediante SPSS, adoptando p <0,05. En total, los participantes eran predominantemente estudiantes $(94,6 \%, \mathrm{n}=3166)$, con una mediana de edad de 21 años. La prevalencia del vegetarianismo fue mayor entre los estudiantes de cursos de áreas distintas a la salud $(6,8 \%)$ y entre las mujeres $(7,2 \%)$, con $\mathrm{p}<0,001$. Entre los vegetarianos $(\mathrm{n}=196)$, hubo un predomínio de la clase lacto-ovo vegetariana $(63,8 \% ; \mathrm{n}=125)$ y los veganos representaron el $10,2 \%(\mathrm{n}=20)$. El motivo ético fue el más mencionado entre los adherentes $(50,5 \%)$ y la mayoría refirió buscar información sobre la práctica en fuentes no científicas como sítios de internet $(74,4 \%, \mathrm{n}=134)$ y blogs $(25,6 \%, \mathrm{n}=46)$. La puntuación de conocimientos en nutrición osciló entre 6 y 14, con un promedio de 11,7 $\pm 1,52$ y mediana de 12,0 puntos, mayor para las mujeres $(11,94 \pm 1,33, \mathrm{p}=0,006)$. Conocer el perfil de los vegetarianos es fundamental para satisfacer las necesidades de este público.
\end{abstract}

Palabras clave: Alimentación colectiva; Nutrición; Vegetarianismo.

\title{
1. Introdução
}

A alimentação humana é influenciada por diversos fatores, de ordens culturais, religiosas, sociais, ambientais, econômicas e pessoais (Brasil, 2014; Pula, Parks, \& Ross, 2014). No contexto histórico mundial, a revolução industrial foi um marco importante nas modificações dos hábitos alimentares da população (Pellerano, 2017). Tal processo foi o principal responsável pela urbanização, industrialização e inserção das mulheres no mercado de trabalho e, juntamente com a melhoria na educação e na qualidade de vida da população, estão entre os principais motivos do aumento no número de refeições realizadas fora do lar nas últimas décadas, em diferentes países, inclusive no Brasil (Pellerano, 2017; Instituto Brasileiro de Geografia e Estatística, 2019). Consequentemente, houve expansão e aumento do número de restaurantes comerciais e institucionais, também conhecidos como Unidades Produtoras de Refeições (Oliveira \& Silva, 2015).

No Brasil, o setor responsável pelo fornecimento de alimentação dentro das Universidades é o Restaurante Universitário (RU), o qual é regulamentado pelo Programa Nacional de Assistência Estudantil (PNAES), cujo objetivo é melhorar as condições de permanência dos estudantes no ambiente universitário, reduzindo os índices de evasão e minimizando a desigualdade social (Imperatori, 2017).

A universidade é um ambiente que concentra indivíduos com culturas e hábitos diversificados, dentre os quais destaca-se o vegetarianismo (França, 2017). Tal prática é comumente definida como aquela que exclui, parcialmente ou totalmente, os produtos de origem animal da alimentação, sendo denominada de veganismo quando essa exclusão está relacionada também ao vestuário e outras esferas de consumo (Sociedade Vegetariana Brasileira, 2021). A identificação dos adeptos pode ser realizada com base em diferentes critérios e, ainda, considerando o autorrelato.

Diversas motivações levam os indivíduos a abster-se do consumo de proteína de origem animal e essas geralmente estão relacionadas aos aspectos éticos, de saúde e ambientais, além da influência dos meios de comunicação, sobretudo da internet (Petti et al., 2017). Considerando a recente difusão e tendência crescente da prática vegetariana entre populações e grupos como os universitários (Fonseca et al., 2021), ressalta-se a escassez de estudos com este público e a necessidade de mais informações que permitam que profissionais de saúde atendam aos vegetarianos de forma mais satisfatória.

Diante do exposto, esse estudo tem como objetivo avaliar o perfil sociodemográfico, de hábitos alimentares e de conhecimentos em nutrição dos clientes vegetarianos de um Restaurante Universitário. 


\section{Metodologia}

Trata-se de um estudo transversal (Bedaque \& Bezerra, 2018) realizado com clientes de um estabelecimento de alimentação coletiva, vinculado a uma instituição de ensino superior localizada na cidade de Vitória-ES. A pesquisa foi aprovada pelo Comitê de Ética em Pesquisa com Seres Humanos do Centro de Ciências da Saúde/Universidade Federal do Espírito Santo, sob o número CAAE 50915115.0.0000.5060.

Foram entrevistados 3348 clientes que frequentaram o restaurante durante os dias úteis do mês de março/2016, nos horários em que eram servidas as refeições almoço e jantar. Os clientes foram abordados na fila e após serem esclarecidos dos objetivos e etapas da pesquisa, assinaram o Termo de Consentimento Livre e Esclarecido, autorizando sua participação no estudo. Aplicou-se um questionário semiestruturado com questões sobre perfil sociodemográfico e hábitos alimentares, adaptado dos estudos de Guadagnin (2010) e Leite (2004) e complementado por informações da Sociedade Vegetariana Brasileira (2021). Foi realizado um estudo piloto com 20 clientes de dois estabelecimentos voltados para o público de vegetarianos para avaliar a adequação do instrumento. Após este teste, o questionário foi modificado e posteriormente utilizado no presente estudo.

A entrevista foi conduzida de forma individual e voluntária, com duração média de cinco minutos para os clientes que não eram vegetarianos e de dez minutos para que os fossem identificados como tal. As questões sociodemográficas e de hábitos alimentares foram respondidas por todos os participantes, sendo a seção de frequência de consumo de proteína animal utilizada para a identificação/classificação dos vegetarianos. Adotou-se o critério de classificação proposto nos estudos da Universidade Loma Linda que considera como não-vegetariano: aquele indivíduo que consome carne vermelha, aves, peixe, ovos, leite e derivados $\geq 1 \mathrm{x}$ semana; vegetariano: consome todos os alimentos de origem animal < 1 x mês; semi-vegetariano: consome carne vermelha, aves, peixe $\geq 1 \times$ mês e $<1 \times$ semana, sendo o consumo de ovos, leite e derivados livre; ovolactovegetariano: consome carne vermelha, aves e peixes <1 x mês, ovos, leite e derivados $\geq 1$ x mês; pescovegetariano: consome de carne vermelha e aves $<1$ x mês, peixe $\geq 1$ x mês, sendo o consumo de ovos e leite livre; e vegano: ausência de consumo de alimentos e utilização de qualquer produto de origem animal (Rizzo, Sabaté, Jaceldo-Siegl, \& Fraser, 2011; Tonstad et al., 2013).

Os clientes classificados como vegetarianos responderam questões sobre a prática do vegetarianismo e de conhecimentos em nutrição, por meio de questionário adaptado de Guadagnin (2010). Tal questionário apresentava uma lista com 14 alimentos e questões relacionadas à presença de muito ou pouco sal e gordura nos mesmos. Para cada acerto nas respostas foi atribuído um ponto e realizou-se o somatório da pontuação total para cada participante, podendo variar de 0 a 14 .

Os dados foram armazenados no Microsoft Excel e analisados no software Statistical Package for the Social Sciences versão 21.0. As variáveis categóricas foram apresentadas em forma de porcentagem (\%) e para análise de associação foi utilizado o teste de Qui-quadrado. As variáveis não categóricas foram apresentadas em forma de média \pm desvio padrão, mediana (mínimo e máximo) e para testar a normalidade foi utilizado o Teste de Kolmogorov-Smirnov. Para comparar as medidas de tendência central das variáveis não paramétricas foram utilizados os testes de Mann-Whitney e Kruskall-Wallis. Para todos os testes foi adotado o nível de significância de $\mathrm{p}<0,05$.

\section{Resultados}

Os participantes do estudo eram predominantemente universitários $(94,6 \%, \mathrm{n}=3166)$ e apresentaram mediana de idade de 21 anos, variando de 18 a 76 anos (Tabela 1). Do total, 6,5\% (n=218) se autodeclararam vegetarianos, porém ao considerar a classificação a partir do consumo alimentar e o critério adotado, a prevalência do vegetarianismo foi de 5,9\% (n=196.

Verificou-se que ser do sexo feminino e estudante de cursos diferentes da área da saúde associaram-se ao vegetarianismo $(\mathrm{p}<0,001)$ (Tabela 1). A idade dos adeptos se mostrou superior aos demais ( $\mathrm{p}=0,011)$. Além disso, 
diferentemente dos demais clientes, os vegetarianos relataram consumir habitualmente os pratos sem carne ofertados e com maior frequência $(\mathrm{p}<0,001)$ (Tabela 1$)$.

Tabela 1: Perfil sociodemográfico e hábito alimentar de clientes de um restaurante universitário. Vitória, ES, 2016.

\begin{tabular}{|c|c|c|c|c|}
\hline Características & $\begin{array}{c}\text { Vegetariano }^{b} \\
196(5,9)\end{array}$ & $\begin{array}{c}\text { Não } \\
\text { Vegetariano }^{b} \\
3152(94,1)\end{array}$ & $\begin{array}{c}\text { Total }^{b} \\
3348(100,0)\end{array}$ & $p$-valor \\
\hline${\text { Idade }(\operatorname{anos})^{\mathrm{a}}}$ & $22,0(18-55)$ & $21,0(18-76)$ & $21,0(18-76)$ & $0,011^{c}$ \\
\hline \multicolumn{5}{|l|}{ Campus $^{b}$} \\
\hline Ciências da Saúde & $32(3,4)$ & $897(96,6)$ & $929(27,7)$ & \multirow[t]{2}{*}{$<0,001^{d}$} \\
\hline Demais Áreas & $164(6,8)$ & $2255(93,2)$ & $2419(72,3)$ & \\
\hline \multicolumn{5}{|l|}{$\operatorname{Sexo}^{b}$} \\
\hline Feminino & $121(7,2)$ & $1557(92,8)$ & $1678(50,1)$ & \multirow{2}{*}{$<0,001^{d}$} \\
\hline Masculino & $75(4,5)$ & $1595(95,5)$ & $1670(49,9)$ & \\
\hline \multicolumn{5}{|l|}{ Profissão $^{b}$} \\
\hline Estudante & $187(5,9)$ & $2979(94,1)$ & $3166(94,6)$ & \multirow{4}{*}{$0,692^{d}$} \\
\hline Técnico Administrativo & $6(6,6)$ & $85(93,4)$ & $91(2,7)$ & \\
\hline Professor & $1(2,1)$ & $47(97,9)$ & $48(1,4)$ & \\
\hline Outros & $2(4,7)$ & $41(95,3)$ & $43(1,3)$ & \\
\hline \multicolumn{5}{|l|}{ restaurante universitáriob } \\
\hline Almoço & $92(4,8)$ & $1805(95,2)$ & $1897(56,7)$ & \multirow{3}{*}{$<0,001^{d}$} \\
\hline Jantar & $13(3,7)$ & $335(96,3)$ & $348(10,4)$ & \\
\hline Almoço e Jantar & $91(8,3)$ & $1012(91,7)$ & $1103(32,9)$ & \\
\hline \multicolumn{5}{|l|}{ Consumo do prato vegetariano $^{b}$} \\
\hline Sim & $186(8,5)$ & $2010(91,5)$ & $2196(65,6)$ & \multirow{2}{*}{$<0,001^{d}$} \\
\hline Não & $10(0,9)$ & $1142(99,1)$ & $1152(34,4)$ & \\
\hline \multicolumn{5}{|c|}{$\begin{array}{l}\text { Frequência do consumo do prato } \\
\text { vegetariano }^{b}\end{array}$} \\
\hline Esporadicamente & $5(0,8)$ & $605(99,2)$ & $610(27,8)$ & \multirow{4}{*}{$<0,001^{d}$} \\
\hline Semanalmente & $10(2,3)$ & $417(97,7)$ & $427(19,4)$ & \\
\hline 2 a 4 x por semana & $48(5,7)$ & $794(94,3)$ & $842(38,4)$ & \\
\hline Diariamente & $123(38,8)$ & $194(61,2)$ & $317(14,4)$ & \\
\hline
\end{tabular}

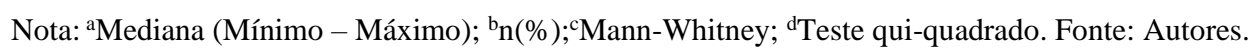

A classe vegetariana mais identificada foi a de ovolactovegetarianos $(63,8 \%, n=125)$ e os veganos representaram $10,2 \%(n=20)$, sendo observada associação com a procura por orientação profissional $(\mathrm{p}=0,004)$ (Tabela 2). O nutricionista foi o principal profissional mencionado pelos participantes $(77,9 \%, \mathrm{n}=53)$. Além disso, a maioria dos participantes declarou buscar informações sobre alimentação vegetariana $(92,3 \%, n=180)$ (Tabela 2) e as principais fontes citadas foram sites na internet $(74,4 \%, \mathrm{n}=134)$, blogs $(25,6 \%, \mathrm{n}=46)$ e livros $(18,9 \%, \mathrm{n}=34)$ (dados não demonstrados em tabelas). 
Tabela 2: Associação entre o tipo de vegetarianismo o perfil dos clientes vegetarianos de um restaurante universitário. Vitória, ES, 2016.

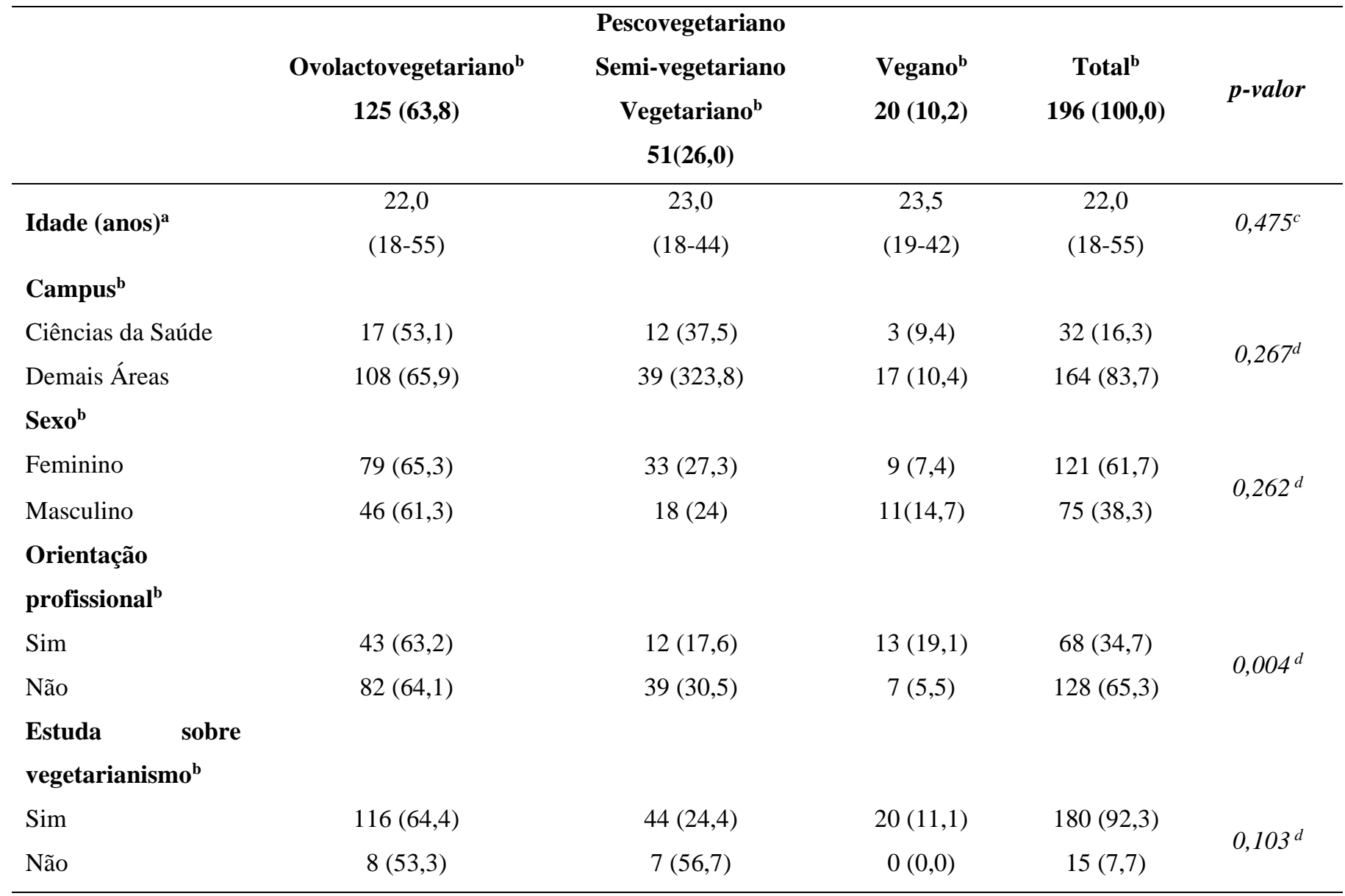

Nota: ${ }^{a}$ Mediana (Mínimo - Máximo); ${ }^{\mathrm{b}}$ (\%); ${ }^{\mathrm{c} K r u s k a l-W a l l i s ; ~}{ }^{\mathrm{d}}$ Teste qui-quadrado. Fonte: Autores.

Entre os principais motivos que levaram à adoção do vegetarianismo pelos entrevistados estavam a ética (50,5\%, $\mathrm{n}=99)$, a saúde $(33,7 \%, \mathrm{n}=66)$ e outros como paladar e a influência da mídia $(39,9 \%, \mathrm{n}=78)$. Foi encontrada diferença no tempo de vegetarianismo e os motivos éticos $(\mathrm{p}=0,002)$, ambiental $(\mathrm{p}=0,003)$ e outros $(\mathrm{p}=0,003)$ (Tabela 3). Além disso, o motivo saúde foi associado a não procura por orientação profissional ( $\mathrm{p}=0,012)$ e o motivo "outros" se associou ao sexo feminino $(\mathrm{p}=0,040)$ (Tabela 3$)$.

Tabela 3: Associação entre o motivo pela adoção do vegetarianismo e o perfil dos clientes vegetarianos de um restaurante universitário.

\begin{tabular}{lcccc}
\hline & Ético & Saúde & Ambiental & Outros $^{\mathbf{c}}$ \\
\hline Idade (anos) $^{\mathbf{a}}$ & $21,0(18-43)$ & $24,0(18-44)$ & $21,0(18-38)$ & $23,0(18-55)$ \\
-valor $^{d}$ & 0,010 & 0,465 & 0,551 & 0,132 \\
Campus $^{\mathbf{b}}$ & & & & \\
Ciências da Saúde & $13(13,1)$ & $15(22,7)$ & $5(13,9)$ & $14(17,9)$ \\
Demais Áreas & $86(86,9)$ & $51(77,3)$ & $31(86,1)$ & $64(82,1)$ \\
-valor $^{d}$ & 0,221 & 0,084 & 0,661 & 0,617 \\
Sexo $^{\mathbf{b}}$ & & & & \\
\hline
\end{tabular}


Research, Society and Development, v. 10, n. 15, e345101522850, 2021

(CC BY 4.0) | ISSN 2525-3409 | DOI: http://dx.doi.org/10.33448/rsd-v10i15.22850

\begin{tabular}{|c|c|c|c|c|c|}
\hline Feminino & & $61(61,6)$ & $37(56,1)$ & $20(55,6)$ & $55(70,5)$ \\
\hline Masculino & & $38(38,4)$ & $29(43,9)$ & $16(44,4)$ & $23(29,5)$ \\
\hline$p$-valor ${ }^{d}$ & & 0,972 & 0,244 & 0,399 & 0,040 \\
\hline \multicolumn{6}{|c|}{ Orientação profissional $^{\mathrm{b}}$} \\
\hline Sim & & $33(33,3)$ & $15(22,7)$ & $13(36,1)$ & $33(42,3)$ \\
\hline Não & & $66(66,7)$ & $51(77,3)$ & $23(63,9)$ & $45(57,7)$ \\
\hline$p$-valor ${ }^{d}$ & & 0,686 & 0,012 & 0,843 & 0,069 \\
\hline \multicolumn{6}{|c|}{ vegetarianismo $^{\text {b }}$} \\
\hline Sim & & $95(96,0)$ & $58(89,2)$ & $32(91,4)$ & $71(91,0)$ \\
\hline Não & & $4(4,0)$ & $7(10,8)$ & $3(8,6)$ & $7(9,0)$ \\
\hline$p$-valor ${ }^{d}$ & & 0,052 & 0,254 & 0,829 & 0,583 \\
\hline $\begin{array}{l}\text { Tempo } \\
\text { vegetarian }\end{array}$ & $\begin{array}{l}\text { de } \\
n o s)^{a}\end{array}$ & $2,0(0,08-19)$ & $3,0(0,08-16)$ & $1,0(0,08-38)$ & $4,0(0,08-34)$ \\
\hline$p$-valor ${ }^{e}$ & & 0,002 & 0,260 & 0,003 & 0,003 \\
\hline
\end{tabular}

Nota: ${ }^{a}$ Mediana (Mínimo - Máximo); ${ }^{b}$ (\%); ${ }^{c}$ OUTROS: Religião, Economia, Família, Curso, Filosofia, Paladar, Político, Ideologia e Mídia; ${ }^{\mathrm{d} Q u i-q u a d r a d o ;}{ }^{\mathrm{e}}$ Mann-Whitney. Fonte: Autores.

A pontuação referente ao conhecimento em nutrição variou de 6 a 14, com média 11,7 $\pm 1,52$ e mediana de 12,0. Foi evidenciada maior média de pontos para o sexo feminino $(11,94 \pm 1,33, \mathrm{p}=0,006)$, porém não houve diferença ao considerar o campus $(p=0,945)$, busca por orientação profissional $(p=0,387)$ e estudos sobre o tema $(p=0,399)$. Além disso, observou-se que a idade não apresentou correlação com a referida pontuação $(\mathrm{p}=0,575)$ (dados não demonstrados em tabelas).

\section{Discussão}

Neste estudo encontrou-se que a prevalência do vegetarianismo entre universitários foi de 5,9\% ( $\mathrm{n}=196)$, porém 6,5\% $(n=218)$ se autodeclararam vegetarianos. Ambos os percentuais foram superiores ao encontrado em pesquisa realizada com 15453 indivíduos dos Estados Unidos entre 2007-2010, em que 2,1\% (n=323) se autoidentificaram vegetarianos, sendo 68\% do sexo feminino e apresentaram média de idade 35,3 anos (Juan, Yaminia, \& Brittenb, 2015). Apesar do presente estudo também ter identificado maioria de vegetarianos do sexo feminino, a média de idade encontrada foi menor do que na pesquisa supracitada cujo valor foi de 23,7 anos (Juan, Yaminia, \& Brittenb, 2015), possivelmente em função do público universitário se caracterizar majoritariamente por jovens.

No Brasil, resultados de uma consulta pública realizada no ano de 2018 demonstraram que $14 \%$ dos entrevistados se autodeclararam vegetarianos (Instituto Brasileiro de Opinião Pública e Estatística, 2018). Em outra pesquisa realizada com 583 indivíduos na Universidade de São Paulo, onde os mesmos foram questionados sobre o que pensam em relação à carne e quanto à prática vegetariana, encontrou-se que 3,9\% eram vegetarianos, sendo que $62 \%$ eram mulheres com média de idade de 21,3 anos (Ruby et al., 2016).

Quanto ao motivo de adesão à alimentação vegetariana identificados nesta pesquisa, destaca-se a ética, a qual envolve os direitos dos animais. De forma semelhante, Hargreaves et al. (2020) em pesquisa realizada com 5014 vegetarianos brasileiros identificou que 60,5\% mencionaram a ética/moral como principal motivação para a prática vegetariana. No geral, as pessoas que se tornam vegetarianas por razões éticas, consideram essenciais os aspectos morais, emocionais e filosóficos para o bem-estar dos animais (Petti et al., 2017; Ruby et al., 2016). 
No presente estudo, outra razão importante para adesão ao vegetarianismo foi a saúde, visto que foi relatada por $33,7 \%$ ( $\mathrm{n}=66)$ dos adeptos. Tal achado se assemelha ao encontrado por Hargreaves et al. (2020), onde 11,3\% dos participantes relataram questões ligadas à saúde pessoal como principal motivação. Neste sentido, vale ressaltar a publicação da Organização Mundial da Saúde (2015) que adverte sobre a relação entre o consumo de carnes processadas e o aumento de incidência de doenças como o câncer.

No que diz respeito à obtenção de informações sobre a prática vegetariana, apesar da maioria dos participantes relatar a realização de estudos sobre o vegetarianismo, as fontes mais mencionadas foram a literatura não científica como sites da internet e blogs. Mesmo assim, especula-se que esta procura por informações possa ter influenciado os resultados de conhecimentos em nutrição, cuja média de acertos encontrada foi alta $(11,7 \pm 1,52)$. Tais acertos foram superiores para o sexo feminino o que pode ser reflexo do maior interesse das mulheres em assuntos relacionados à alimentação, nutrição e peso corporal, especialmente durante a fase universitária (Yahia et al., 2016).

Em relação à procura por suporte profissional, resultados semelhantes ao presente estudo foram observados por Meneguci et al. (2021) que realizaram pesquisa com 116 universitários de uma faculdade particular do sul de Minas Gerais e encontraram que a maioria não fazia acompanhamento nutricional, sendo esse mencionado apenas pelos ovolactovegarianos $(46,15 \%)$. Os autores identificaram que as baixas ingestões de gordura saturada e de colesterol foram mencionadas como os principais benefícios do vegetarianismo por $65,7 \%$ dos ovolactovegetarianos, $100 \%$ dos vegetarianos estritos e dos veganos entrevistados.

\section{Considerações Finais}

$\mathrm{O}$ perfil de adeptos ao vegetarianismo encontrado neste estudo foi predominantemente feminino, jovem e ovolactovegetarianos, cuja motivação de adesão mais citada foi a ética. Entre os que buscavam suporte profissional, houve destaque para o papel do nutricionista, porém, a maioria mencionou procurar por informações relacionadas à prática vegetariana em fontes não científicas. Houve variação no nível de conhecimento sobre nutrição entre os participantes do estudo e maiores pontuações foram identificadas para o sexo feminino.

Os achados desse estudo permitem conhecer melhor o público vegetariano universitário, porém ressalta-se a necessidade de outras pesquisas sobre a temática, bem como propostas de novas preparações para serem inseridas em cardápios de unidade produtoras de refeições, a fim de atender as necessidades e as expectativas desse público.

\section{Referências}

Bedaque, H. P. \& Bezerra, E. L. M. (2018). Descomplicando MBE: uma abordagem prática da medicina Baseada em evidências. Caule de Papiro.

Brasil (2014). Guia alimentar para a população brasileira. In Ministério da Saúde. Secretaria de Atenção Primária à Saude, Departamento de Atenção Básica.

Fonseca, J. F. A., Marques, M. A. A; Campagnaro, L. B.; Santos, L. N.; Pereira, L. R.; Martinez, O. G. E.; Silva, D. A (2021). Análise comparativa da satisfação do cardápio e aceitabilidade de preparações vegetarianas em restaurante universitário. Research, Society and Development, 10 (12), e44101220109. http://dx.doi.org/10.33448/rsd-v10i12.20109

França, J. F. M. (2017). Refeições vegan em ambiente escolar: Lei n. ${ }^{\circ} 11 / 2017$ e as suas implicações em Unidades de Alimentação e Nutrição. Faculdade de Ciências da Nutrição e Alimentação da Universidade do Porto. https://repositorioaberto.up.pt/bitstream/10216/107054/2/210281.pdf.

Guadagnin, S. C. (2010). Elaboração e validação de Questionário de Conhecimentos em Nutrição para Adultos. http://repositorio.unb.br/bitstream/10482/7204/1/2010_SimoneCostaGuadagnin.pdf.

Hargreaves, S.M., Nakano, E.Y. \& Zandonadi, R,P (2020). Brazilian Vegetarian Population-Influence of Type of Diet, Motivation and Sociodemographic Variables on Quality of Life Measured by Specific Tool (VEGQOL). Nutrients, 12(5):1406. https://doi.org/10.3390/nu12051406

Imperatori, T. K. (2017). A trajetória da assistência estudantil na educação superior brasileira. Serviço Social \& Sociedade. 129: 285-303. https://doi.org/10.1590/0101-6628.109

Instituto Brasileiro de Geografia e Estatística. (2019). Pesquisa de orçamentos familiares 2017-2019: análise do consumo alimentar pessoal no Brasil. https://biblioteca.ibge.gov.br/visualizacao/livros/liv101670.pdf 
Research, Society and Development, v. 10, n. 15, e345101522850, 2021

(CC BY 4.0) | ISSN 2525-3409 | DOI: http://dx.doi.org/10.33448/rsd-v10i15.22850

Instituto Brasileiro de Opinião Pública e Estatística. (2018). Pesquisa de Opinião Pública Sobre Vegetarianismo. IBOPE Inteligência, 1-24.

Juan, W., Yaminia, S., \& Brittenb, P. (2015). Food Intake Patterns of Self-identified Vegetarians among the U.S. Population, 2007-2010.Procedia Food Science, 4, 86-93. https://doi.org/10.1016/j.profoo.2015.06.013.

Leite, M. L. (2004). Demonstrar o perfil da população que frequenta os restaurantes vegetarianos da Asa Norte, Brasília - DF. http://bdm.unb.br/bitstream/10483/557/1/2004_MileneLuccasLeite.pdf.

Meneguci, R.; Silvério, A.P.; Bazuco, G.T.; Santis, G.M.; Merotti, I.N.; Silvério, A.C.P. (2021). Conhecimento sobre os aspectos nutricionais que envolvem a dieta vegetariana. Revista Científica Multidisciplinar, 2 (9): e29719. https://doi.org/10.47820/recima21.v2i9.719

Oliveira, T. C. \& Silva, D.A. (2015). Administração de unidade produtoras de refeições. Rubio.

Pellerano, J. A. (2017). Industrialização e alimentação: Impactos da Revolução Industrial moderna em produção,distribuição, preparo e consumo de alimentos. Anais da VI Reunião de Antropologia da Ciência e Tecnologia. https://ocs.ige.unicamp.br/ojs/react/article/view/2764/2625

Petti, A., Palmieri, B., Vadala, M., \& Laurino, C. (2017). Vegetarianism and veganism: not only benefits but also gaps. A review. Progress in Nutrition, 19(3), 229-242. https://doi.org/10.23751/pn.v19i3.5229

Pula, K., Parks, C. D., \& Ross, C. F. (2014). Regulatory focus and food choice motives. Prevention orientation associated with mood, convenience, and familiarity. Appetite, 78C, 15-22. https://doi.org/10.1016/j.appet.2014.02.015

Rizzo, N. S., Sabaté, J., Jaceldo-Siegl, K., \& Fraser, G.E. (2011). Vegetarian dietary patterns are associated with a lower risk of metabolic syndrome: the Adventist health study 2. Diabetes Care, 34 (5), 1225-1227. https://doi.org/10.2337/dc10-1221

Ruby, M. B., Alvarenga, M. S., Rozin, P., Kirby, T. A., Richer, E., Rutsztein, G. (2016). Attitudes toward beef and vegetarians in Argentina, Brazil, France, and the USA. Appetite, 96, 546-554. https://doi.org/10.1016/j.appet.2015.10.018

Sociedade Vegetariana Brasileira. (2021).Vegetarianismo. https://www.svb.org.br/vegetarianismo1/o-que-e

Tonstad, S., Stewart, K., Oda, K., Batech, M., Herring, R.P., Fraser, G.E. (2013). Vegetarian diets and incidence of diabetes in the Adventist Health Study-2. Nutrition Metabolism \& Cardiovascular Diseases, 23 (4), 292-299. https://doi.org/doi:10.1016/j.numecd.2011.07.004

World Health Organization. (2015). IARC Monographs evaluate consumption of red meat and processed meat. Lyon: International agency for research on cancer. http://www.iarc.fr/en/media-centre/pr/2015/pdfs/pr240_E.pdf

Yahia, N., Brown, C.A., Rapley, M., \& Chung, M. (2016). Level of nutrition knowledge and its association with fat consumption among college students. BMC Public Health, 16 (1), 1-10. https://doi.org/10. doi:10.1186/s12889-016-3728-z 